\title{
Capillary Freezing or Complete Wetting of Hard Spheres in a Planar Hard Slit?
}

\author{
Marjolein Dijkstra \\ Debye Institute, Soft Condensed Matter, Utrecht University, Princetonplein 5, 3584 CC Utrecht, The Netherlands
}

(Received 7 November 2003; published 2 September 2004)

\begin{abstract}
Extensive simulations of a hard sphere fluid confined between two planar hard walls show the onset of crystalline layers at the walls at about $98.3 \%$ of bulk crystallization density $\rho_{f}$ independent of the wall separations $L_{z}$, and is, hence, a single wall phenomenon. As the bulk density far from the wall $\rho_{b}$ increases, the thickness of the crystalline film appears to increase logarithmically, with $\left(\rho_{f}-\rho_{b}\right)$ indicating complete wetting by the hard sphere crystal of the wall-fluid interface. Increasing $\rho_{b}$ further, we observe a jump in the adsorption which depends on $L_{z}$ and corresponds to capillary freezing. The formation of crystalline layers below bulk crystallization, the logarithmic growth of the crystalline film, its independence of $L_{z}$, and its clear distinction from capillary freezing lend strong evidence for complete wetting by the hard sphere crystal at the wall-fluid interface.
\end{abstract}

DOI: 10.1103/PhysRevLett.93.108303

The theory of simple fluids is often based on the hardsphere system, which serves as a standard reference system for determining the structure and phase behavior of more complicated fluids. The hard-sphere system has been studied in great detail and its bulk phase behavior is now well understood. In particular, it was shown by computer simulations that a system of pure hard spheres shows a purely entropy-driven transition from a disordered fluid phase to a face-centered-cubic (fcc) crystal phase [1] at sufficiently high density. The state of affairs for the interfacial behavior of hard spheres near a planar hard wall is less clear cut. Such a system plays a similar (reference) role for simple fluids, colloids, or colloidpolymer mixtures in contact with substrates. The behavior of fluids in contact with substrates plays an important role in areas of wetting, adhesion, and heterogeneous nucleation. A contentious issue, which has received much attention, is whether or not there is complete wetting by the hard-sphere crystal at a smooth hard wall. The main reason for this interest resides in the fact that any complete wetting is driven by entropy alone. The discussion was instigated in 1992 by Courtemanche and Van Swol, whose molecular-dynamics simulations of hard spheres confined between two hard walls showed spontaneous formation of crystalline layers near the wall [2] at a normal pressure below the saturated bulk freezing pressure. The authors concluded that there is complete wetting by the hard-sphere crystal, also called prefreezing, at the wall-fluid interface. However, these findings are disputed by others as the presence of crystalline layers can be due to metastability, equilibration problems (density profiles are very asymmetric and vacancies are found in the wetting crystal) [2], finite size effects [3], or capillary freezing [4]. Indeed simulations with fixed normal pressure show that the onset of the formation of crystalline layers at the wall shifts to higher bulk densities and approaches the value at which bulk crystallization occurs upon increasing the plate separation [3]. On the other hand, it is claimed in Ref. [4] that the prefreezing of
PACS numbers: 82.70.Dd, 64.60.Cn, 64.70.-p, 68.08.Bc

hard spheres as found in the simulations [2] is a manifestation of capillary freezing, i.e., confinement induced freezing of the whole fluid rather than the formation of crystalline layers at the wall. Using the Kelvin equation, the capillary freezing is predicted to occur at $98.5 \%$ of bulk crystallization for the plate separation used in Ref. [2], while prefreezing is found at $98.6 \%$ of bulk crystallization. Finally, recent computer simulations show [5] that the height of the nucleation barrier for a crystal nucleus attached to a planar hard wall is about 2 orders of magnitude lower than for the homogeneous system, but the barrier does not disappear completely. The presence of a nonzero nucleation barrier is usually considered incompatible with the complete wetting scenario. Also Groot et al. argued that a system of hard spheres should not show a tendency to crystallize at the wall as the density of the fluid layer in contact with the wall does not reach the two-dimensional hard-disc freezing density when one approaches bulk coexistence [6].

It therefore remains an open question as to whether or not complete wetting by the hard-sphere crystal occurs at the smooth hard wall-fluid interface. In this Letter, we provide evidence for complete wetting based on extensive computer simulations.

In principle, the complete wetting scenario can be confirmed by calculating the contact angle $\theta$, defined by $\cos \theta=\left(\gamma_{w f}-\gamma_{w s}\right) / \gamma_{f s}$, where $\gamma_{w f}, \gamma_{w s}$, and $\gamma_{f s}$ are, respectively, the interfacial tension between the wall-fluid phase at a bulk density $\rho_{b}=\rho_{f}$, the wall-crystal phase at $\rho_{b}=\rho_{s}$, and the solid-fluid interface at bulk coexistence. The densities of the coexisting bulk fluid and bulk solid phase of hard spheres are $\rho_{f} \sigma^{3}=0.943$ and $\rho_{s} \sigma^{3}=$ 1.041, respectively [1]. A vanishing contact angle corresponds to complete wetting of the wall-fluid interface by the crystal phase. One might suppose that this issue could be settled by calculating interfacial tensions using density functional theory. However, these calculations are very sensitive to the precise details of the functional and they are complicated by the difficulty in describing the sym- 
metry and rapid spatial variations in the density for the solid and solid-fluid interface. Using a full free minimization of the density functional based on a weighted density approximation, Ohnesorge et al. calculated the three interfacial tensions and found that $\cos \theta=1.15$ [7]. They conclude that the wall is wetted completely by the solid phase with the (111) axis normal to the wall. However, the theoretical absolute values of the interfacial tensions $\left|\gamma_{w f}\right|$ and $\left|\gamma_{w s}\right|$ are about $60 \%$ above the absolute values calculated recently in simulations[8] and the theoretical $\gamma_{f s}$ is $50 \%$ smaller than found in simulations [9]. A free minimization study of the recent fundamental measure theory, which is known to be more accurate for describing the bulk [10], is lacking. Using the interfacial tensions from simulations $[8,9]$, we find $\cos \theta=0.98 \pm$ 0.36 . Thus we are unable to draw any definite conclusions given the statistical accuracy of the existing results.

In order to investigate the adsorption and, in particular, the wetting properties of a fluid, it is important to study a bulk fluid in contact with a single wall. This single-wall system is difficult to treat in simulations, since the fluid in contact with the wall at $z=0$ cannot be treated with periodic boundary conditions in the $z$ direction. Recently, a new simulation method was developed for treating such a system [11]. A fluid is simulated under the condition that the density far from the wall reaches a bulk density $\rho_{b}$ by imposing a penalty function which suppresses large deviations from $\rho_{b}$. Applying this technique to the present system allowed us to study only the onset of prefreezing, as we were not able to find a penalty function that suppresses the density fluctuations from $\rho_{b}$ adequately when we approach bulk coexistence. Note that the prefreezing found in previous simulations [2] does not occur until a few percent below bulk crystallization. Density fluctuations by more than a percent at a bulk density that is just below bulk crystallization results in additional surface transitions, which should be avoided.

In many simulations of adsorption phenomena, the fluid is confined between two identical walls in the $x y$ plane, located at $z=0$ and $z=L_{z}$. The additional wall at $z=$ $L_{z}$ should not affect the adsorption of the fluid at the wall of interest at $z=0$ provided $L_{z}$ is sufficiently large that there is a flat portion of the density profile in the central region of the slit. A drawback of using large $L_{z}$ is that long simulation times are needed which may hamper a systematic study of the growth of a thick wetting film. However, using smaller values of $L_{z}$ can lead to finite size (capillary) effects and these may hinder the observation of the single-wall phenomena of interest.

In this Letter, we take advantage of the competition between capillary effects and single-wall (wetting) phenomena in planar slits. By making a systematic study of the effect of plate separation, we were able to distinguish a regime where complete wetting of the hard-sphere crystal appears to occur, which is a single-wall phenomenon (i.e., independent of plate separation), from the phe- nomenon of capillary freezing, which depends on plate separation.

We perform extensive Monte Carlo simulations of pure hard spheres with a diameter $\sigma$ confined between two planar hard walls at different wall separations $L_{z}$. In many previous simulation studies, the external pressure normal to the walls $P_{z}$ is kept constant. This requires very long equilibration times due to volume fluctuations. Moreover, for finite $L_{z}, P_{z}$ is not directly related to a bulk pressure. We therefore decided to perform our simulations in the canonical ensemble, i.e., we fix the number of particles $N$ and the volume $V=L_{x} L_{y} L_{z}$. The temperature $T$ is an irrelevant quantity for systems with only hard-core interactions. Our initial configuration is a facecentered-cubic crystal at a density $\rho_{s}$ with the (111) axis normal to the walls. Simulations of wall-induced crystal nucleation show that the first particles that crystallize on a smooth hard wall form a (111) plane with a lattice spacing that is commensurate with a bulk density $\rho_{s}$ [5]. Moreover, complete wetting by the hard-sphere crystal can only occur by the coexisting bulk crystal with density $\rho_{s}$. Transverse dimensions (and dimensions in the $z$-direction) that are incommensurate with a bulk crystal at density $\rho_{s}$ result into stresses in the solid. In that case, we expect that the wetting scenario shifts towards higher densities or that complete wetting will disappear. The transverse dimensions of the simulation box are $L_{x}=$ $8.86 \sigma$ and $L_{y}=9.59 \sigma$ and the second wall is at $L_{z}=$ $21.8 \sigma, L_{z}=43.5 \sigma, L_{z}=65.21 \sigma$, and $L_{z}=86.91 \sigma$. We also performed simulations for a system with $L_{x}=$ $13.29 \sigma, L_{y}=13.43 \sigma$, and $L_{z}=43.5 \sigma$ in order to check the system size dependence and we found good agreement with the results obtained from the smaller system. The number of particles $N$ in our initial configurations are, respectively, $N=1920,3840,5760$, and 7680 . We perform simulations at different bulk densities $\rho_{b}<\rho_{f}$ by removing particles in our initial configuration, while keeping the box dimensions fixed. Here $\rho_{b}$ is determined from the flat central part of the measured density distribution $\rho(z)$. Typically, $10^{7}-10^{8}$ Monte Carlo sweeps were needed for equilibration, and the profiles were accumulated in bins of width $\delta z=0.01 \sigma$ over a further $10^{7}$ sweeps (one sweep is one attempted move per particle).

Figure 1 shows the density profiles $\rho(z)$ for $L_{z}=43.5 \sigma$ at varying bulk densities $\rho_{b}$. At the top, the density profile of a fcc crystal with $\rho_{b} \sigma^{3}=1.041$ and the (111) axis normal to the walls is shown, while the density profile at the bottom is that of a liquid with $\rho_{b} \sigma^{3}=$ $0.9246 \pm 0.0005$. The two density profiles in the two middle figures indicate the formation of crystalline layers at the wall clearly: $\rho(z)$ drops to zero between the density peaks close to the walls. The density profile in the central region of the slit is flat and can be associated with a bulk fluid at $\rho_{b} \sigma^{3}=0.938 \pm 0.002$ and $0.935 \pm 0.001$, respectively. We clearly observe that the thickness of the portion of the film with crystalline ordering increases with 


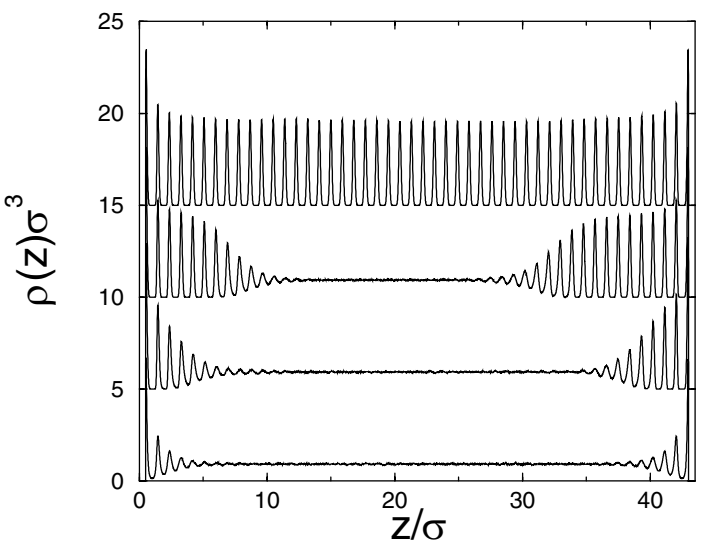

FIG. 1. Density profiles $\rho(z)$ of hard spheres between two planar hard walls at separation $L_{z}=43.5 \sigma$, from which we determine the bulk densities $\rho_{b} \sigma^{3}=1.041,0.938,0.935$, and 0.9246 from top to bottom.

increasing $\rho_{b}$. The growth of the thickness of the crystalline film can also be appreciated by calculating the adsorption $\Gamma \sigma^{2}=\left(N-\rho_{b} V\right) \sigma^{2} /\left(L_{x} L_{y}\right)$ for varying $\rho_{b}$. Figure 2 shows $\Gamma$ as a function of $\rho_{b}$ for four values of $L_{z}$. We observe that the adsorption increases when $\rho_{b}$ approaches $\rho_{f}$ (denoted by the vertical dashed line) in agreement with Ref.[2]. The error bar for $\Gamma$ can be obtained from the error in $\rho_{b}$. For comparison, we also plot an empirical fit obtained from simulations for the adsorption of a hard-sphere fluid at a hard wall [12]; the latter was made for values of $\rho_{b} \sigma^{3}$ well-removed from $\rho_{f}$. For $\rho_{b} \sigma^{3} \geq 0.925$, the adsorption deviates from this fit due to the formation of crystalline layers at the wall. In the inset, we show the adsorption as a function of $\rho_{b}$ obtained from our single-wall simulations using the penalty function. We

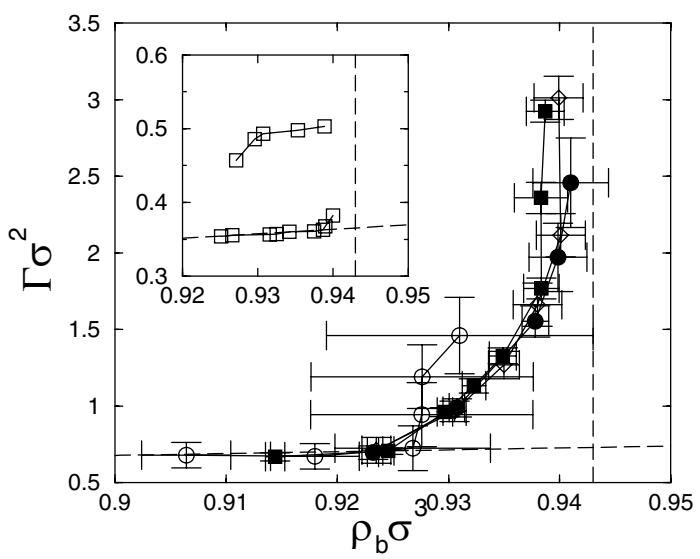

FIG. 2. The adsorption $\Gamma$ of a fluid of hard spheres in a planar slit versus the bulk density $\rho_{b}$ for wall separations $L_{z} / \sigma=$ 21.8(○), 43.5(ם), 65.21( $\diamond), 86.91(\bigcirc)$. The near horizontal dashed line denotes an empirical fit to $\Gamma$ obtained from earlier simulations [12] at values of $\rho_{b}$ well-below the bulk transition at $\rho_{f}$ (vertical line). The near vertical portion of $\Gamma$ correspond to capillary freezing. The inset shows a jump in $\Gamma$ corresponding to the crystallization of the first fluid layer obtained from single-wall simulations. Note the pronounced hysteresis. see a clear jump in the adsorption corresponding to a firstorder phase transition. Calculating the in-plane correlation functions we can associate the lower branch with a system where all the layers adsorbed to the wall are fluidlike, while the upper branch corresponds to a system where the first layer at the wall is frozen. Consequently, the crystallization of the first layer is discontinuous in accordance with the observation of a nucleation barrier of a crystal nucleus of hard spheres (which appears to be very flat and about one layer thick) at a smooth hard wall [5]. Unfortunately, we were not able to study whether the subsequent layers freeze in a first-order or continuous fashion. Nevertheless, the form of the density profiles and the increased adsorption as a function of $\rho_{b}$ lend strong support for complete wetting by the hard-sphere crystal in the limit $\rho_{b} \rightarrow \rho_{f}$. More quantitative support for the complete wetting scenario is provided by the measured logarithmic increase of the adsorption $\Gamma$ as a function of $\left(\rho_{f}-\rho_{b}\right) / \rho_{f}$. Figure 3 shows that the adsorption curves consist of three regimes: (i) At $\rho_{b} \sigma^{3} \lesssim 0.925$ we find that $\Gamma$ is close to the empirical fit for the adsorption obtained in Ref. [12]. (ii) At large $\rho_{b}$, we find a vertical rise in the adsorption, which we associate with capillary freezing induced by the presence of two walls. The bulk density $\rho_{c}$ at which this jump occurs increases with wall separation and approaches $\rho_{f}$ from below. We plot $\rho_{c}$ as a function of $L_{z}^{-1}$ in Fig. 4. We see that $\rho_{c}$ decreases significantly with decreasing $L_{z}$. In the limit $L_{z} \rightarrow \infty$ the capillary freezing density $\rho_{c}$ approaches bulk freezing $\rho_{f}$ linearly in $L_{z}^{-1}$. This linear limiting behavior can be derived from the Kelvin equation, which reads

$$
\beta\left(\mu_{\text {coex }}-\mu_{c}\right)=\frac{2 \beta\left(\gamma_{w f}-\gamma_{w s}\right)}{\left(\rho_{s}-\rho_{f}\right) L_{z}}
$$

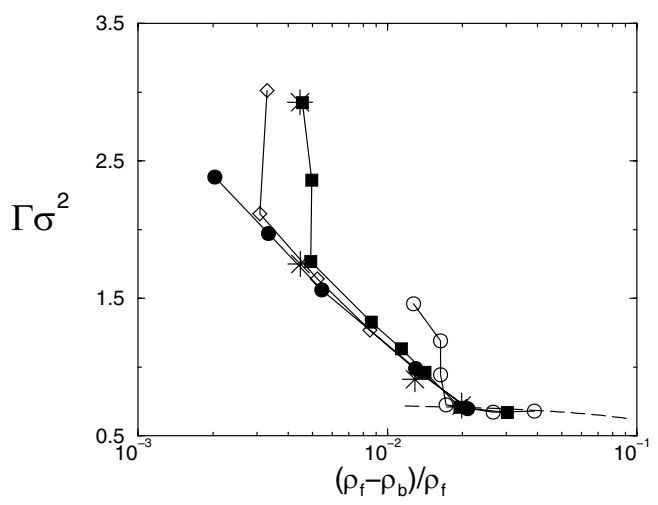

FIG. 3. The adsorption $\Gamma$ of a fluid of hard spheres in a planar slit versus $\left(\rho_{f}-\rho_{b}\right) / \rho_{f}$ for four values of $L_{z}$. The symbols are as in Fig. 2. For $\rho_{b} \sigma^{3}<0.925$, the adsorption is close to the empirical fit [12]. The vertical rise in $\Gamma$ found at large $\rho_{b}$ corresponds to capillary freezing. For intermediate $\rho_{b}$ the thickness of the wetting crystalline film grows logarithmically. The asterisks are results for $L_{x}=13.29 \sigma, L_{y}=13.43 \sigma$, and $L_{z}=43.5 \sigma$ and confirm there is negligible finite system size dependence. 


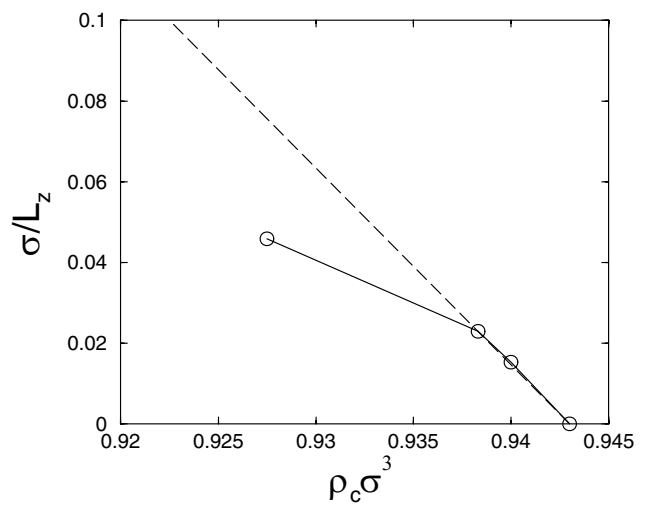

FIG. 4. Capillary freezing density $\rho_{c}$ as a function of $L_{z}^{-1}$, obtained from the vertical rise of $\Gamma$ observed in Fig. 3. The dashed straight line is the prediction of the Kelvin Eq. (1).

where $\mu_{\text {coex }}$ and $\mu_{c}$ are the chemical potentials at bulk coexistence and at capillary freezing, respectively. For sufficiently large $L_{z}$, we can expand the left hand side of Eq. (1) about $\rho_{f}$. Using the Carnahan-Starling expression for the chemical potential of the bulk fluid, we arrive at $\left.\left(\partial \beta \mu / \partial \rho_{b} \sigma^{3}\right)\right|_{\rho_{f} \sigma^{3}}=208.15$ with $\rho_{f} \sigma^{3}=0.943$. Combining this result with Eq. (1) and using $\rho_{s} \sigma^{3}=1.041$, and the interfacial tensions obtained from simulations [3], i.e., $\beta \gamma_{w f} \sigma^{2}=-3.80 \pm 0.18$ and $\beta \gamma_{w s} \sigma^{2}=-4.37 \pm$ 0.10 , we find $\left(\rho_{f}-\rho_{c}\right) \sigma^{3}=0.2051 \sigma / L_{z}$, denoted by the dashed line in Fig. 4. For $L_{z} / \sigma=43.5,65.21$ we find that the capillary freezing is predicted accurately by the Kelvin equation. For $L_{z}=86.91 \sigma$, the Kelvin equation predicts that capillary freezing should occur at $\left(\rho_{f}-\right.$ $\left.\rho_{b}\right) / \rho_{f} \simeq 0.85 \times 10^{-3}$. However, we were not able to reach this regime as the statistical errors of $\rho_{b}$ and $\rho_{f}$ are now of the same order. (iii) For $0.925 \lessgtr \rho_{b} \sigma^{3} \lesssim$ $\rho_{c} \sigma^{3}$, we observe that $\Gamma$ increases logarithmically as $\rho_{b} \rightarrow \rho_{c}$. The adsorption can be fitted by $\Gamma \sigma^{2}=A_{1}+$ $A_{2} \ln \left[\left(\rho_{f}-\rho_{b}\right) / \rho_{f}\right]$, with fit parameters $A_{1}=-2.2 \pm 0.2$ and $A_{2}=-0.73 \pm 0.04$. We recall that general arguments for complete wetting for systems with short-ranged forces imply the wetting film thickness $l$ should diverge as $-\xi_{b} \ln \left[\left(\rho_{f}-\rho_{b}\right) / \rho_{f}\right]$ where $\xi_{b}$ is the bulk correlation length of the wetting phase in this case the crystal. For very thick films $l / \sigma=\Gamma /\left[2\left(\rho_{s}-\rho_{f}\right) \sigma\right]$ so that $\left|A_{2}\right| \simeq$ $2 \xi_{b}\left(\rho_{s}-\rho_{f}\right) \sigma^{2}$, implying $\xi_{b} / \sigma \simeq 3.8 \pm 0.2$. Note that we have divided the adsorption by two as $\Gamma$ is defined for two walls. We clearly see that all the adsorption curves in Fig. 3 lie close to each other for $\rho_{b}<\rho_{c}$. We also observe from Fig. 3 that the formation of crystalline layers and the logarithmic growth of the film thickness both start at $\left(\rho_{f}-\rho_{b}\right) / \rho_{f} \simeq 0.017$, i.e., $98.3 \%$ of bulk crystallization independent of plate separation, and can, hence, both be interpreted as a single-wall phenomenon. However, we were only able to follow the logarithmic growth in a small density regime, i.e., $10^{-3} \lesssim\left(\rho_{f}-\rho_{b}\right)$ / $\rho_{f} \lesssim 10^{-2}$ and one could argue that the logarithmic growth might cease just before bulk coexistence, due to the presence of stresses. However, it is hard to believe that stresses can arise in a hard-sphere crystal which is grown at a smooth planar wall. Earlier studies for a (111) patterned surface show that complete wetting of the hardsphere crystal already sets in at $29 \%$ of bulk crystallization [13]. It is tempting to argue that in the present case the adsorbed crystalline layers at the wall act as a template which induces crystallization, resulting in complete wetting in a similar fashion to a static patterned surface.

In conclusion, we provide strong evidence for complete wetting by the hard-sphere crystal of the wall-fluid interface as our simulations show the formation of crystalline layers at the wall at $98.3 \%$ of bulk crystallization and the logarithmic growth of the crystalline film, which are both independent of plate separation (single-wall phenomenon) and well separated from capillary freezing that does depend on plate separation. The interfacial behavior of hard spheres near a hard wall serves as a good model for colloidal hard spheres and can play a reference role for more complicated fluids. It is interesting to study the effect of size polydispersity and of nonadsorbing polymer, giving rise to depletion interactions, on the interfacial behavior. This effect will be the focus of future work.

It is a pleasure to thank R. van Roij, M. Schmidt, and R. Evans for stimulating discussions.

[1] W.W. Wood and J. D. Jacobson, J. Chem. Phys. 27, 1207 (1957); B. J. Alder and T. E. Wainwright, J. Chem. Phys. 27, 1208 (1957); W. G. Hoover and F. H. Ree, J. Chem. Phys. 49, 3609 (1968).

[2] D. J. Courtemanche and F. van Swol, Phys. Rev. Lett. 69, 2078 (1992); D. J. Courtemanche, T. A. Pasmore, and F. van Swol, Mol. Phys. 80, 861 (1993).

[3] M. Heni, Ph.D. thesis, Heinrich Heine Universität Düsseldorf. 2001.

[4] W. K. Kegel, J. Chem. Phys. 115, 6538 (2001).

[5] S. Auer and D. Frenkel, Phys. Rev. Lett. 91, 015703 (2003).

[6] R. D. Groot, N. M. Faber, and J. P. van der Eerden, Mol. Phys. 62, 861 (1987); R. D. Groot, M. Elwenspoek, and P. Bennema, J. Cryst. Growth 79, 817 (1986).

[7] R. Ohnesorge, H. Löwen, and H. Wagner, Phys. Rev. E 50, 4801 (1994).

[8] M. Heni and H. Löwen, Phys. Rev. E 60, 7057 (1999).

[9] R. L. Davidchack and B. B. Laird, Phys. Rev. Lett. 85, 4751 (2000).

[10] R. Roth, R. Evans, A. Lang, and G. Kahl, J. Phys. Condens. Matter 14, 12063 (2002), and references therein.

[11] M. Dijkstra, R. van Roij, and R. Evans, Phys. Rev. E 63, 051703 (2001).

[12] J. R. Henderson and F. van Swol, Mol. Phys. 51, 991 (1984).

[13] M. Heni and H. Löwen, Phys. Rev. Lett. 85, 3668 (2000). 